\title{
Inter-laboratory measurement comparison between INRIM and ESA on electrical quantities
}

\author{
Flavio Galliana ${ }^{1, *}$, Pier Paolo Capra $^{2}$, Roberto Cerri ${ }^{3}$, Vincenzo D’Elia ${ }^{4}$,Enrico Gasparotto ${ }^{5}$ Luca Roncaglione Tet $^{6}$, \\ Marco Del Moro ${ }^{7}$, and Alessandro Cozzani ${ }^{8}$ \\ ${ }^{1,2,3,4,5,6}$ National Institute of Metrological Research, strada delle Cacce 91, 10135 Turin, Italy \\ ${ }^{7,8}$ Serco Nederland BV for ESA - European Space Agency, Keplerlaan 1, 2201 AZ Noordwijk, The Netherlands
}

\begin{abstract}
Inter-laboratory comparisons (ILCs) are an effective mean to establish the compatibility of the measurements among laboratories. As consequence they are a mean to assure confidence in the competence and in the correctness of the dissemination processes from national Standards in different countries. This paper refers on a ILC between the National Institute of Metrological Research (INRIM-Italy) and the European space research and technology Centre (ESA- ESTEC), belonging to two different countries, on DC Voltage, DC Resistance and Electrical Capacitance carried out in 2016. The comparison showed a satisfactory agreement between the results of the two Laboratories.
\end{abstract}

\section{Introduction}

Today, most technical products, especially in aerospatial sectors, are developed in more countries. For this reason, to assure that the various parts of the products can be correctly assembled, the production must be under control and the measurements made in different countries must be compatible. Every country has its own measurement system normally involving a National Metrology Institute (NMI) a network of calibration laboratories and a larger number of organizations for which the traceability of the measuring devices to national measurement standards has to be assured. The inter-laboratory comparisons (ILCs) are the most widely used mean to compare the results of the measurements carried out by calibration laboratories. They are also important to monitor the quality of calibration results according to [1]. ILCs can be carried out among NMIs as in [2-4]. Another kind of ILCs involves a NMI and secondary Laboratories of the same country accredited or not, so belonging to the same measurement system. The ILC reference values of these comparisons are the measurement values given by the NMI that is normally at an upper metrological level with respect the other Laboratories [5-8]. In this paper an ILC, made according to the EN ISO/IEC 17043 international Standard [9] between the National Institute of Metrological Research (INRIM) and the European space research and technology Centre (ESA- ESTEC) on DC Voltage, DC Resistance and Electrical Capacitance is presented.
The National Institute of Metrological Research (INRIM) is the Italian National Metrology Institute. It realizes, maintains, improve and compare with other NMIs measurement standards for the majority of SI units. It carries out research focused on metrology and innovative technologies, disseminates and transfers scientific results, technology and know-how to scientific, industrial and service users. It participates in research programs and activities, also collaborating with universities and other public and private entities. The European Space Agency (ESA) coordinates the European space programs, develops space technologies and services. ESA also works with space organizations outside Europe. The European Space Research and Technology Centre (ESTEC) develops and co-ordinates most ESA projects on science, exploration, telecommunications, human spaceflight, satellite navigation and Earth observation. In ESTEC operates a metrology laboratory performing mechanical, opto-mechanical and dimensional measurements for the ESTEC Test Centre, ESA laboratories and ESA projects. The Laboratory has primary electrical standards traceable to national and international standards. The Laboratory is ISO 17025 accredited for DC voltage, resistance, electrical capacitance, temperature/humidity by the Dutch Accreditation Council RvA. This Laboratory made the measurements concerning the ILC described in the paper.

\section{INRIM and ESA-ESTEC}

\footnotetext{
Corresponding author: f.galliana@inrim.it
}

C The Authors, published by EDP Sciences. This is an open access article distributed under the terms of the Creative Commons Attribution License 4.0 (http://creativecommons.org/licenses/by/4.0/). 


\section{Standards to be calibrated in the ILC}

The traveling standard for DC Voltage was a J. Fluke mod. 732 B Reference standard to be calibrated at $1.018 \mathrm{~V}$ and $10 \mathrm{~V}$ (see Fig. 1). The traveling standards for DC Resistance were respectively: a J. Fluke model 742A-1 $\Omega$ (Fig. 2), a $10 \Omega$ Leeds \& Northrup mod. 4030-B. (Fig. 3) and a $1 \mathrm{M} \Omega$ Leeds \& Northrup mod. 4050-B. (Fig. 4) standard resistors. The traveling standard for electrical capacitance was an ESI mod. SC1000 $1000 \mathrm{pF}$ standard capacitor to be calibrated at $1 \mathrm{kHz}$ (Fig. 5).

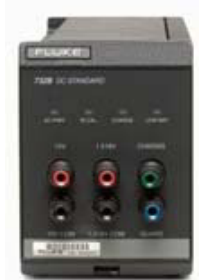

Fig.1. J. Fluke mod. 732 B DC Voltage standard.

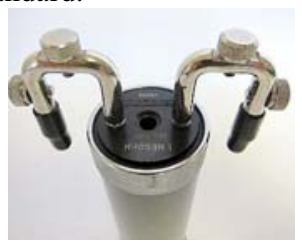

Fig. 4. $1 \mathrm{M} \Omega \mathrm{L} \& \mathrm{~N} 4050-\mathrm{B}$.

Fig. 5. 1000 pF ESI SC1000.

\subsection{Measurements instructions for the ILC}

The calibrations had to be performed at the following conditions: laboratory temperature of $23.0{ }^{\circ} \mathrm{C} \pm 1{ }^{\circ} \mathrm{C}$ (or better). The Fluke mod. 732B DC Voltage standard had to be calibrated at $1.018 \mathrm{~V}$ and $10 \mathrm{~V}$. It had to be supplied with a sinusoidal AC power supply with RMS voltage of $230 \mathrm{~V} \pm 5 \%$ (or better) and frequency $50 \mathrm{~Hz}$ $\pm 1 \%$. The power supply had to be applied $48 \mathrm{~h}$ beforeThe measurements had to be started after $1 \mathrm{~h}$ since the switching the instrument supply from mains to its rechargeable batteries. In these conditions, the standard outputs (at $1.018 \mathrm{~V}$ and $10 \mathrm{~V}$ ), the Rt values provided by the instrument and the measurement time for each output had to be reported.

The $1 \Omega$ and $10 \Omega$ resistors had to be placed in a stable oil bath controlled at a temperature of $23.0^{\circ} \mathrm{C}$ in order to insure a short-term instability of $0.1{ }^{\circ} \mathrm{C}$ or better, or in free air at a of $23.0 \pm 0.3{ }^{\circ} \mathrm{C}$ and in four-terminal configuration at with preferably currents of $30 \mathrm{~mA}$ and $100 \mathrm{~mA}$ for the $1 \Omega$ and $10 \mathrm{~mA}$ and $30 \mathrm{~mA}$ for the $10 \Omega$. The resistors had to be connected with shielded cables. The $1 \mathrm{M} \Omega$ resistor had to be placed in free air or in an air bath. It had to be calibrated at $10 \mathrm{~V}$ in four terminal configuration.

The calibration of the standard capacitor, equipped with two coaxial connectors $\mathrm{H}$ and $\mathrm{L}$ with the external conductor of the $\mathrm{H}$ terminal insulated while that of the $\mathrm{L}$ terminal connected to the shield, had to be performed with the external conductor of the $\mathrm{H}$ and $\mathrm{L}$ terminals shorted together with the inner conductor of the L terminal set at the shield potential, but electricallyinsulated from it and with a capacitor power supply of $1.5 \mathrm{~V}$ at $1 \mathrm{kHz}$. In these conditions, the capacitance between the $\mathrm{H}$ and $\mathrm{L}$ terminals had to be determined.

\subsection{Reference values}

As ILC reference values were considered the mean values of the INRIM calibrations obtained before and after the calibration at the ESA-ESTEC laboratory. The measurement results along with their uncertainties of the two laboratories were reported in regular calibration certificates according to their calibration procedures respectively approved by INRIM as signatory of the CIPM MRA ${ }^{\mathrm{a}}$ and by the Dutch Accreditation Council RvA.

\section{Analysis of the results}

The ILC results were evaluated by means of the normalized error En, according to [9]. Therefore, the INRIM reference values (as mean of two calibrations also to evaluate the stability of the traveling standards) and the values of the ESA-ESTEC laboratory were respectively defined as:

$$
\begin{aligned}
& m_{I} \pm U_{I} \\
& m_{L} \pm U_{L}
\end{aligned}
$$

where $U_{I}$ and $U_{L}$ are the corresponding expanded uncertainties, respectively. From $U_{I}$ and $U_{L}$ the standard uncertainties were obtained: $u_{I} \cong \frac{1}{2} U_{I}$ and $u_{L} \cong \frac{1}{2} U_{L}$ The following differences were then calculated:

$$
y=m_{L}-m_{I}
$$

whose standard uncertainties are:

$$
u_{y}^{2}=\left[u_{L}^{2}+u_{I}^{2}-2 u_{L} u_{I} \times r\left(m_{L}, m_{I}\right)\right]
$$

where $r\left(m_{L}, m_{I}\right)$ is the correlation coefficient between the measurements at INRIM and at the ESA-ESTEC laboratory. In this case $r=0$ as the measurements carried out by the two laboratories were independent as belonging to two different countries and the ESAESTEC reference Standards are calibrated at the Dutch NMI, VSL.

Finally, the normalized error $E_{n}$ was evaluated as:

$$
E_{n}=\frac{y}{U_{y}}
$$

\footnotetext{
a The CIPM Mutual Recognition Arrangement (CIPM MRA) is the framework through which National Metrology Institutes demonstrate the international equivalence of their measurement standards and the calibration and measurement certificates they issue. The outcomes of the Arrangement are the internationally recognized (peer-reviewed and approved) Calibration and Measurement Capabilities (CMCs) of the participating institutes.
} 
An ILC result is considered satisfactory if $|E n| \leq 1$. Normally, when in an ILC are involved an NMI and calibration Laboratories belonging to the same country, it is important to correctly evaluate the possible correlation between the measurements of the NMI and the secondary Laboratories as normally the Laboratories send their reference standards to the NMI for calibration. Suggestion for this evaluation can be found in $[1,5-7,10]$. The ILC results are reported in Tables 1-3

Table 1. Results of the ILC for DC Resistance.

\begin{tabular}{|c|c|c|c|c|c|c|c|}
\hline$(\Omega)$ & $m_{I}(\Omega)$ & $U\left(m_{I}\right)(\mu \Omega)$ & $m_{L}(\Omega)$ & $U\left(m_{L}\right)(\mu \Omega)$ & $y(\mu \Omega)$ & $U(y))(\mu \Omega)$ & $E_{n}$ \\
\hline $1^{\mathrm{b}}$ & 1.00001115 & 1.0 & 1.000003 & 45 & -8.1 & 45 & -0.2 \\
\hline $10^{\mathrm{c}}$ & 10.0004365 & 10 & 10.000444 & 450 & 7.5 & 450.0 & 0.0 \\
\hline$(\mathrm{M} \Omega)$ & $(\mathrm{M} \Omega)$ & $(\Omega)$ & $(\mathrm{M} \Omega)$ & $(\Omega)$ & $(\Omega)$ & $(\Omega)$ & \\
\hline $1 \mathrm{M}^{\mathrm{d}}$ & 1.00004955 & 3.0 & 1.000044 & 30 & -5.6 & 30.1 & -0.2 \\
\hline
\end{tabular}

Table 2. Results of the ILC for DC Voltage.

\begin{tabular}{|c|c|c|c|c|c|c|c|}
\hline$(\mathrm{V})$ & $m_{I}(\mathrm{~V})$ & $U\left(m_{I}\right)(\mu \mathrm{V})$ & $\left.m_{L}\right)$ & $U\left(m_{L}\right)(\mu \mathrm{V})$ & $y(\mu \mathrm{V})$ & $U(y)(\mu \mathrm{V})$ & $E_{n}$ \\
\hline 1.018 & 1.0181286 & 3.1 & 1.018129 & 5.1 & 0.4 & 5.8 & 0.1 \\
\hline 10 & 10.0000279 & 5.0 & 10.00003 & 50 & 0.2 & 50 & 0.0 \\
\hline
\end{tabular}

Table 3. Results of the ILC for electrical Capacitance.

\begin{tabular}{|c|c|c|c|c|c|c|c|}
\hline$(\mathrm{pF})$ & $m_{I}(\mathrm{pF})$ & $U\left(m_{I}\right)(\mathrm{fF})$ & $m_{L}(\mathrm{pF})$ & $\left.U\left(m_{L}\right) \mathrm{fF}\right)$ & $y(\mathrm{fF})$ & $U(y)(\mathrm{fF})$ & $E_{n}$ \\
\hline 1000 & 1000.04915 & 7.0 & 1000.047 & 10 & -2.2 & 12.2 & -0.2 \\
\hline
\end{tabular}

${ }^{\mathrm{b}}$ The laboratory made the measurement at $10 \mathrm{~mA}$. This value was compared with the INRIM measurement at $30 \mathrm{~mA}$.

${ }^{c}$ The laboratory made the measurement at $1 \mathrm{~mA}$. This value was compared with the INRIM measurement at $10 \mathrm{~mA}$.

${ }^{\mathrm{d}}$ The laboratory made the measurement at $5 \mathrm{~V}$. This value was compared with the INRIM measurement at $10 \mathrm{~V}$.

In figure 6-8 the same results are reported in graphical way. Uncertainty bars are related to a $2 \sigma$ confidence level.

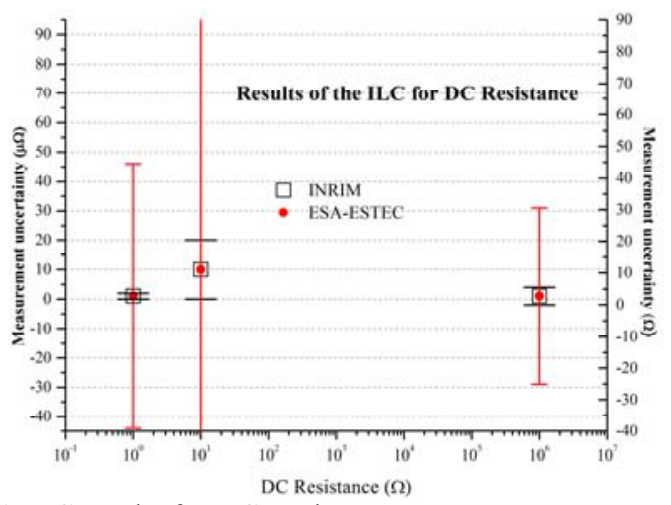

Fig. 6. ILC results for DC Resistance.

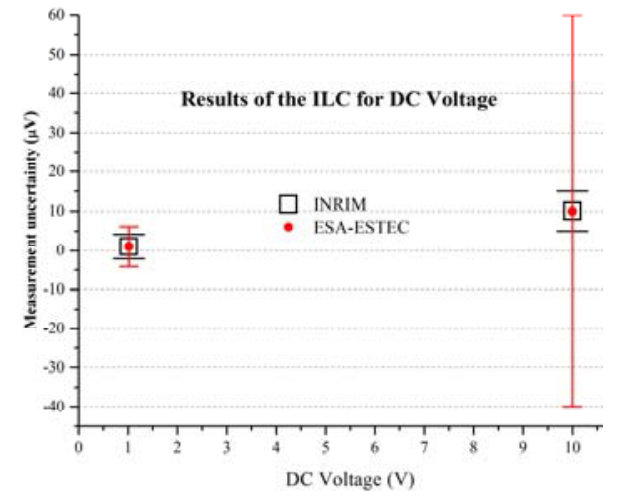

Fig. 7. ILC results for DC Voltage.

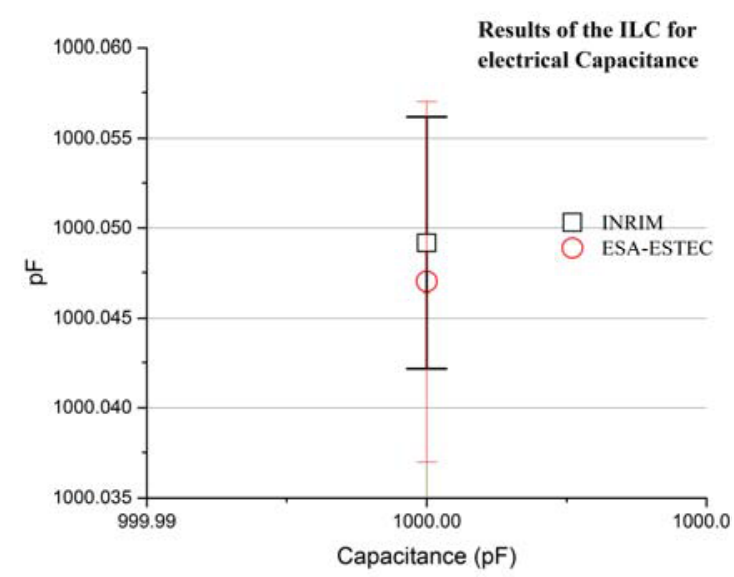

Fig. 8. ILC results for Electrical Capacitance.

\section{Conclusions}

From tables 1 to 3 and from Fig. 6-8 it can be observed that ILC had satisfactory results with extremely low values of the normalized errors $E_{n}$. This result means that the ESA-ESTEC laboratory has adequate competence, instrumentation and calibration procedures to sustain its capabilities as accredited by RvA but also shows the agreement between the measurements of two important scientific institutions belonging to different countries and therefore to different measurement systems. These Institutions play a strategic role in addressing scientific and industrial communities and research programs. In addition, this result is a further demonstration that international agreements such as the CIPM MRA or the 
EA MLA ${ }^{\mathrm{e}}$ are correctly implemented. Future aim of the collaboration between INRIM and ESA could be an ILC involving as traveling Standard a DC Voltage high accuracy multi-value transportable Standard developed at INRIM [11].

\section{References}

1. ISO/IEC17025:2005 "General requirements for the competence of testing and calibration laboratories.

2. C. Aupetit et al. Metrologia 41 Technical Supplement 07002, (2004)

3. R.F. Dziuba and D. G. Jarrett (2002) Metrologia 3901001 (1A) doi:10.1088/00261394/39/1A/1, (2002).

4. B. Jeckelmann, F.Galliana et al, Metrologia, 50, ISSN: 0026-1394, (2013).

5. F. Galliana, P.P.Capra, E. Gasparotto Measurement 42 pp. 1532-1540, (2009).

6. F.Galliana, P.P. Capra, E. Gasparotto Measurement 45 (3) pp. 615-621, (2012).

7. F.Galliana, E. Gasparotto Measurement 52 pp. 64-70, (2014).

8. C. A Hamilton. IEEE Trans. Instrum. Meas. 54 (1), pp. 206-209(2001).

9. EN ISO/IEC 17043:2010 "Conformity assessment- General requirements for proficiency testing".

10. F. Galliana, M. Lanzillotti Measurement 103 pp. 353-360, (2017).

11. F. Galliana, R. Cerri, L. Roncaglione Tet. Measurement 102, pp. 131-137, (2017).

\footnotetext{
e The EA Multilateral Agreement (EA MLA) is a signed agreement between the EA Full Members whereby the signatories recognise and accept the equivalence of the accreditation systems operated by the signing members, and also the reliability of the conformity assessment results provided by conformity assessment bodies accredited by the signing members.
} 